\title{
Factor de la reflectancia bi-cónica en especies vegetales contrastantes: modelación global
}

\section{Bi-conical reflectance factor in contrasting vegetal species: global modeling}

\author{
Fernando Paz ${ }^{1 \ddagger}$, Alejandro Cano², Martín Bolaños ${ }^{1}$, José Chávez', \\ Ma. Isabel Marin' y Enrique Romero ${ }^{3}$
}

\footnotetext{
${ }^{1}$ Colegio de Postgraduados, Campus Monrecillo. Carretera México-Texcoco km 36.5. Montecillo, Texcoco, Edo. de México, México.

* Autor responsable (ferpazpel@gmail.com)

${ }^{2}$ Campo Experimental Edzná, INIFAP. Nhoyaxe 341. 24000 Campeche, Campeche, México.

${ }^{3}$ Centro Nacional de Investigación Disciplinaria en Conservación y Mejoramiento de Ecosistemas Forestales (CENID-COMEF), INIFAP. Av. Progreso 5,

Barrio de Santa Catarina. 04010 Ciudad de México, CDMX, México.
}

\section{RESUMEN}

La clasificación de la vegetación usando información espectral multi-angular ha sido aproximada con esquemas de modelación restringidos o con hipótesis de homogeneidad o estacionariedad. En este trabajo se presenta un esquema de modelación global de los ángulos cenitales y acimutales en la reflectancia de la vegetación, extendiendo los desarrollos al caso de los efectos de cambios en las propiedades ópticas del fondo de la vegetación (suelos) y de la densidad de plantas (follaje). Esta síntesis se realiza en un espacio superior de compactación que depende esencialmente de dos parámetros y engloba todas las variaciones analizadas. El esquema de modelación global desarrollado se aplicó al caso de arreglos de plantas, diferentes densidades de ocho especies con arquetipos contrastantes y suelos desnudos. La modelación se aplicó a las mediciones de factores de reflectancia bi-cónicos, donde los ajustes resultaron óptimos (generalmente con $\mathrm{R}^{2} \geq 0.99 \mathrm{y}$ errores relativos medios menores al 7\%). El análisis de los resultados mostró que la información espectral multi-angular para clasificación de especies, bajo condiciones generalizadas, mantiene todavía problemas de confusión en la discriminación de especies. Esto puede explicarse por la síntesis de análisis realizada de las variaciones asociadas a la discriminación.

Palabras clave: espacios paramétricos, sensores remotos, CCRF, patrones espectrales, plantas leñosas.

Cita recomendada:

Paz, F., A. Cano, M. Bolaños, J. Chávez, Ma. I. Marin y E. Romero. 2018. Factor de la reflectancia bi-cónica en especies vegetales contrastantes: modelación global. Terra Lainoamericana 36: 61-73.

DOI: https://doi.org/10.28940/terra.v36i1.222

\section{SUMMARY}

Classification of vegetation using multi-angular spectral information has been approximated using restricted modeling schemes or with homogeneity or stationarity hypotheses. This paper presents a global modeling scheme of zenith and azimuthal angles in vegetation reflectance, extending the developments to the case of effects of changes on optical properties of vegetation substrates (soils) and plant density (foliage). This synthesis is performed in an upper space of compaction that depends essentially on two parameters and encompasses all the variations analyzed. The global modeling scheme developed was applied to the case of plant arrangements, different densities, of eight species with contrasting archetypes and bare soils. Modeling was applied to measurements of bi-conical reflectance factors, where adjustments were excellent (generally with $\mathrm{R}^{2} \geq 0.99$ and mean relative errors less than $7 \%$ ). Analysis of the results showed that multi-angular spectral information for species classification under generalized conditions still has problems in discriminating species. This can be explained by the analysis synthesis of variations associated with discrimination.

Index words: parametric spaces, remote sensing, $B R D F$, spectral patterns, woody species. 


\section{INTRODUCCIÓN}

La clasificación de la vegetación natural usando sensores remotos se ha explorado desde los inicios de la operación comercial de la tecnología satelital, con sensores remotos en el espectro electromagnético de onda corta. Los resultados obtenidos hasta la fecha no han sido lo suficientemente estables y confiables para usarse en forma operacional sólida.

Últimamente, se ha planteado que la información espectral multi-angular mejora las clasificaciones de la vegetación (Bicheron et al., 1997; Asner, 2000; Diner et al., 1999 y 2005); aunque los resultados, en la práctica, muestran solo algunas mejorías en las clasificaciones (Barnsley et al., 1997; Hyman y Barnsley, 1997; Gerard, 2003), donde la separación de clases de vegetación está fuertemente influenciada por las diferencias a nivel espectral y, en menor medida, a nivel angular.

La información multi-angular asociada a la vegetación, geometría sol-sensor, es la base para el desarrollo de sensores operacionales tales como el POLDER, que tiene hasta 14 ángulos de observación y una resolución espacial de $7 \mathrm{~km}$; el sensor ATSR-2 con 2 ángulos de visión y una resolución de $1 \mathrm{~km} \mathrm{y,}$ el sensor MISR, con 9 ángulos de visión y resolución espacial de $275 \mathrm{~m}$ a $1.1 \mathrm{~km}$. Por el sistema de barrido de la superficie terrestre, los sensores AVHRR y MODIS introducen un efecto simultáneo de geometría sol-sensor y escala (el tamaño de los píxeles se incrementa con el ángulo de visión; esto es, los píxeles que se alejan de la línea orbital ortogonal a la superficie incrementan sus dimensiones producto de vistas oblicuas del sensor). En el otro extremo, el sensor MISR mantiene fijas las dimensiones de los píxeles a través de un muestreo de barrido temporal a lo largo de su línea orbital.

Un enfoque teórico de clasificación de la vegetación, actualmente operativo con sensores MODIS y MISR, es el que desarrolló el grupo de la Universidad de Boston (Lotsch et al., 2000; Zhang et al., 2002a y b), en el que la metodología parte de una clasificación de tipos de vegetación usando propiedades geométricas asociadas a la transferencia de radiación típicas de la mezcla suelo-vegetación, para seis biomas distintos (Myneni et al., 1997). Los biomas, definidos por sus propiedades de transferencia de radiación, se usan para condicionar las extracciones de parámetros biofísicos mediante información espectral (Knyazikhin et al., 1998a y b). En una perspectiva teórica y estadística, Zhang et al. (2002a y b), argumentan que la clasificación de la vegetación con información espectral multi-angular debe basarse en principios de consistencia entre las propiedades de transferencia de radiación de las clases y sus firmas espectrales distintivas, manteniendo fija la resolución espacial (problema de mezclas). Los argumentos de estos autores apoyan la hipótesis de firmas espectrales multi-angulares únicas para las clases de vegetación (los seis biomas definidos por Myneni et al., 1997).

En el presente trabajo se utilizó un esquema de modelación global para analizar los patrones angulares de diferentes tipos de vegetación, con arquetipos contrastantes entre sí, para revisar si la información espectral angular puede diferenciar las clases estudiadas. La modelación sigue una jerarquía de caracterización: ángulos cenitales, ángulos acimutales, efecto del suelo y densidad de plantas. En un primer trabajo se utilizó un modelo de los ángulos cenitales de visión e iluminación y se revisó la factibilidad de discriminar la vegetación, concluyéndose que existía una confusión de clasificación en términos de los efectos del suelo y densidad de plantas. Para poder ratificar las conclusiones, en el presente estudio se desarrolló un modelo global que reduce toda la variabilidad a un espacio de dos parámetros.

\section{MATERIALES Y MÉTODOS}

\section{Modelación Global del Sistema Suelo-Vegetación}

Las reflectancias asociadas a un objeto terrestre varían en función de los ángulos cenitales de iluminación y visión (Bolaños y Paz, 2010) y de la diferencia acimutal (Paz y Medrano, 2015) por lo que requieren modelarse en forma compacta para poder analizar la discriminación entre clases de vegetación.

En los últimos tiempos, los modelos de la función de distribución de la reflectancia bidireccional (BRDF, por sus siglas en inglés) que más se usan, son los basados en kernels (funciones matemáticas) que combinan modelos de medios turbios y de óptica geométrica (Wanner et al., 1995). Los modelos semi-empíricos principales de kernels son lineales (Wanner et al., 1995) y no lineales (Rahman et al., 1993). Un modelo de la BRDF requiere de al menos tres parámetros (Engelson et al., 1996): uno relacionado con la amplitud de la señal, otro que defina la dependencia con respecto a los ángulos cenitales solares y de visión y, uno que tome en cuenta 
las variaciones acimutales. Esto plantea, al menos, el uso de un espacio tri-dimensional para clasificar a la vegetación. En el caso de píxeles individuales de una imagen satelital, los requerimientos de datos de los modelos actuales de la BRDF deben utilizar hipótesis restrictivas (homogeneidad temporal o espacial) para contar con información para caracterizar las BRDF.

La clasificación de la vegetación natural usando modelos de la BRDF se ha realizado a través de los parámetros ajustados en dichos modelos. En el caso de los kernels lineales (isotrópicos, volumétricos y geométricos), los coeficientes ajustados se utilizan para buscar relaciones con la cobertura aérea y estructura (altura, composición, etc.) para diferentes tipos de vegetación (Gerard y North, 1997; Lovell y Graetz, 2002; Gao et al., 2003). Aunque los resultados de estos estudios muestran relaciones congruentes, del tipo de tendencias generales, entre los parámetros de los modelos de kernels lineales de la BRDF y la estructura de la vegetación, presentan confusión en las clasificaciones y errores de estimación altos en las variables asociadas a las clases de vegetación. Con el modelo no lineal de la BRDF de Rahman et al. (1993), Pinty et al. (2002) y Gobron et al. (2002) proponen que el parámetro $\mathrm{k}$ puede usarse para discriminar tipos de vegetación con fondos muy reflectivos (nieve o desierto), de tal manera que la forma de la BRDF se puede caracterizar en función de que sea cóncava $(\mathrm{k}>1)$, convexa $(\mathrm{k}<1)$ o lineal $(\mathrm{k}=1)$.

\section{Modelación del Ángulo Cenital de Iluminación y Visión}

En Paz y Medrano (2015) se planteó el uso de un modelo de los ángulos cenitales de visión e iluminación de un solo parámetro, lo que redujo la complejidad de los esquemas de clasificación de la vegetación.

Si se define el vector de visión $\Omega(\theta \mathrm{v}, \varnothing \mathrm{v})$ y el de iluminación $\Omega^{\prime}(\theta \mathrm{s}, \phi \mathrm{s})$, donde $\theta$ es un ángulo cenital y $\varnothing$ el acimutal, con v como visión y s iluminación solar, el modelo definido por Bolaños y Paz, 2010 está dado por:

$$
\begin{aligned}
& \chi=90-\theta v+\theta s \\
& R n=\ln (R) \cos (\chi) \\
& \chi=a+b R n
\end{aligned}
$$

donde se utiliza un valor de $\mathrm{a}=90$ para obtener un punto adicional y poder estimar $\mathrm{b}$ con un esquema similar al del IRC del modelo de Bolaños et al. (2007). El uso de la función $\cos (\chi)$, como factor multiplicativo de $\mathrm{R}$, permite definir un punto del patrón Rn- $\chi$, ya que $\cos \left(90^{\circ}\right)=0$, por lo que $\mathrm{Rn}=0$ en $\chi=90^{\circ}(\theta \mathrm{v}=\theta \mathrm{s}$; es decir, el plano del Hot Spot). El ángulo $\theta \mathrm{v}$ no tiene signo.

\section{Modelación de la Diferencia Angular Acimutal}

Si se considera la notación de ángulos acimutales positivos, en la dirección contraria a las manecillas del reloj, Paz y Bolaños (2006) ${ }^{1}$ desarrollaron un modelo general de la BRDF que considera al parámetro $b$ de los efectos de los ángulos cenitales de visión e iluminación:

$$
\begin{aligned}
& d \phi^{\prime}=\phi v-\phi s \\
& \text { Si } d \phi \leq 180, d \phi p=d \phi \\
& \text { Si } d f<180, d \phi p=360-d \phi \\
& \text { Si } d \phi p \leq 90, \zeta=d \phi p+\theta s \\
& \text { Si } d \phi p<90, \zeta=d \phi p-\theta s \\
& b n=b \cos (\zeta) \\
& \zeta=90+B(b n)
\end{aligned}
$$

El modelo de las relaciones (1) sigue el mismo esquema que el de las (2), donde $\zeta$ es una variable de posición que hace simétricos los patrones de $b$. Los ángulos cenitales $\theta$ s (Hot Spot) se consideran en término de sus posiciones: plano (semi-hemisferio) de iluminación y sombreado.

De los modelos de las relaciones (1) y (2), es claro que para conocer la BRDF en forma total solo se requiere conocer el parámetro $\mathrm{B}$, lo que simplifica todo el modelo en la forma más compacta posible: un solo parámetro. Esto permite el análisis de las firmas espectrales multi-angulares en forma muy simple.

\section{Modelación del Efecto del Suelo y la Densidad del Follaje}

En la Figura 1 se muestra el espacio espectral R-IRC del patrón temporal de crecimiento de un cultivo de maíz, representado por curvas de igual índice de área

\footnotetext{
${ }^{1}$ Paz, F. y M. Bolaños. 2006. Modelación de la BRDF en la vegetación: reporte final. Colegio de Postgraduados. Reporte Noviembre para AGROASEMEX, 66 p.
} 


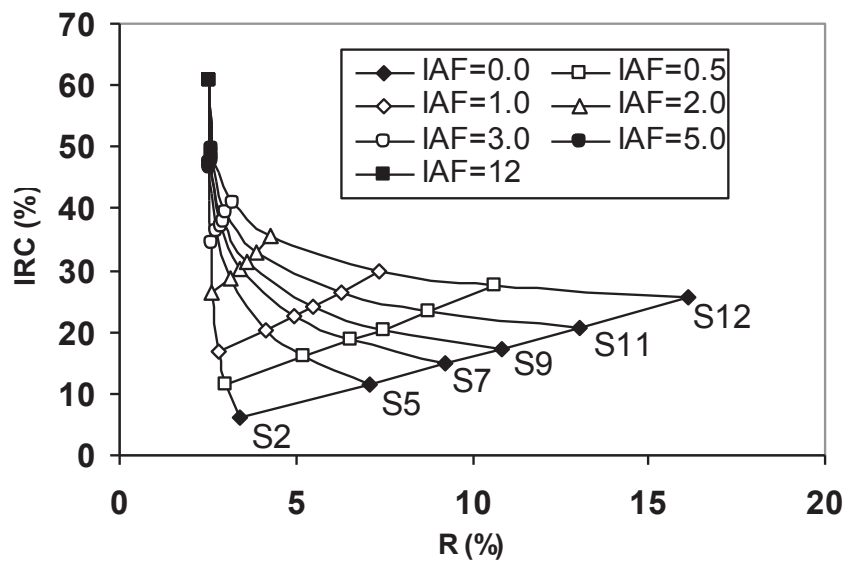

Figura 1. Espacio espectral IRC-R para las simulaciones del cultivo de maíz.

foliar o IAF (iso-IAF). La Figura 1 se generó con el uso de seis tipos de suelos (S2, S5, S7, S9, S11 y S12; del más oscuro al más claro). Paz et al. (2005) detallan las simulaciones radiativas que se muestran en la Figura 1.

El análisis de la Figura 1 define varios patrones muy importantes para entender el comportamiento de la reflectancia durante el desarrollo de los cultivos:

a) $\mathrm{Si}$ se unen los valores de igual IAF (iso-IAF) de cada curva de igual suelo (iso-Suelo), se obtiene un patrón cuasi-lineal.

b) La pendiente e intersección de las líneas rectas de iso-IAF varían con el valor del IAF, como se observa en la Figura 1. La inclinación (pendiente) de las rectas de iso-IAF parte desde una pendiente igual a la de la línea del suelo $(\mathrm{IAF}=0)$ y aumenta hasta alcanzar un ángulo de $90^{\circ}$ en el sentido contrario a las manecillas del reloj. Esta última condición corresponde al caso de saturación de la reflectancia de la banda del $\mathrm{R}$, que se representa en la Figura 1 como los valores de reflectancia arriba del ápice del "sombrero de tres picos" (IAF $>5$ en la Figura 1). Los patrones de los espacios espectrales IRC-Visible (azul, verde y rojo) son similares para todas las bandas del espectro visible, dado que hay una relación lineal entre estas bandas.

c) Todas las curvas de iso-Suelos convergen al mismo punto de saturación de las bandas visibles. En realidad, el sombrero de tres picos tiene una línea recta como ápice, ya que cuando una banda visible se satura, el IRC no lo hace y sigue creciendo hasta su propio punto de saturación. Esta propiedad es importante para el diseño de algoritmos de índices de la señal del suelo y de la vegetación y, refleja la condición física de falta de visibilidad del suelo (relativo a cada banda espectral) y solo se observa la vegetación. El punto de saturación, llamado también de reflectancia infinita o de medio ópticamente denso, es función del espectro de las hojas y de su distribución angular.

Las curvas iso-IAF de la Figura 1 se pueden describir como:

$I R C I_{A F}=a_{0, I A F}+b_{0, I A F} R_{I A F}$

donde el subíndice IAF se refiere a un valor específico del IAF de la mezcla suelo-vegetación. Los parámetros de la recta definida por la ecuación (3), $\mathrm{a}_{0, \mathrm{IAF}}$ (en porcentaje para el caso actual) y $b_{0, I A F}$ (adimensional), dependen del valor de IAF. En lo que sigue se omitirá el subíndice IAF en R e IRC.
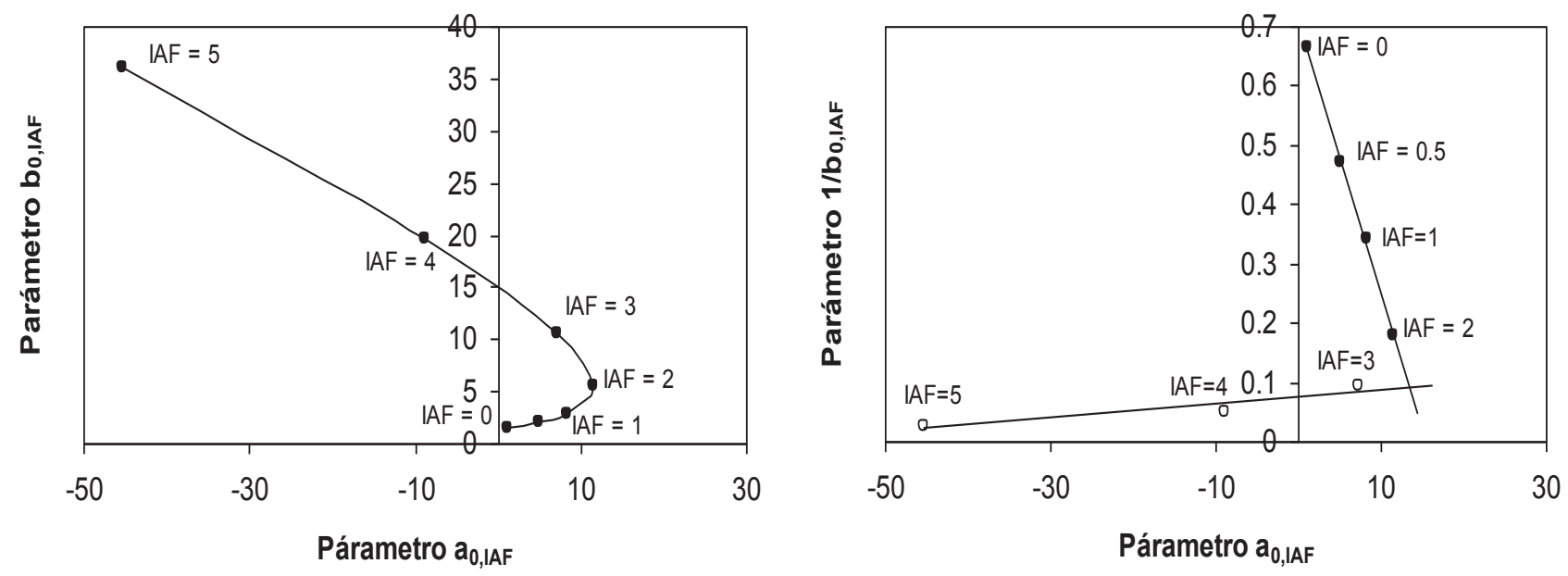

Figura 2. Patrón entre los patrones $a_{0, \mathrm{IAF}}$ y $\mathrm{b}_{0, \mathrm{IAF}}$ de las curvas iso-IAF. 
En la Figura 2 se muestra la relación entre los parámetros $\mathrm{a}_{0, \mathrm{IAF}} \mathrm{y}_{0, \mathrm{IAF}}$ de la Figura 1 (Paz et al., 2007) para el ciclo de crecimiento del cultivo maíz simulado, que va desde el suelo desnudo hasta la condición de cobertura completa del suelo por la vegetación. En la Figura 2 se observa que en la etapa inicial hay un patrón de comportamiento tipo exponencial, hasta un punto donde la banda $\mathrm{R}$ se satura (no cambia de valor). Después del punto de saturación de la banda $\mathrm{R}$, el patrón es del tipo lineal. El punto inicial de la curva $\mathrm{a}_{0, \mathrm{IAF}}-\mathrm{b}_{0, \mathrm{IAF}}$ (Figura 1) representa el caso de suelo desnudo $\left(\mathrm{a}_{0, \mathrm{IAF}=0}=\right.$ $\mathrm{a}_{\mathrm{S}} \mathrm{y}_{0, \mathrm{IAF}=0}=\mathrm{b}_{\mathrm{S}}$ ). El punto donde $\mathrm{a}_{0, \mathrm{IAF}}$ alcanza su valor máximo (punto de transición del patrón exponencial al lineal), representa el final de la fase de crecimiento exponencial y el inicio de la fase lineal. El punto final del patrón lineal de la curva $\mathrm{a}_{0, \mathrm{IAF}}-\mathrm{b}_{0, \mathrm{IAF}}$ representa la situación donde la banda del IRC se satura, lo que ocurre cuando el IAF alcanza su valor máximo. En la misma Figura 2 se muestra una transformación $\left(b_{0, I A F}\right.$ $\rightarrow 1 / b_{0, \mathrm{IAF}}$ ) que hace aproximadamente lineal la función expo-lineal observada. Esta transformación se eligió para modelar en forma adecuada la transición entre la fase exponencial y lineal.

En el caso de la información espectral multiangular, el esquema de modelación propuesto consiste en generar curvas iso-IAF multi-angulares (densidad de plantas o cobertura aérea) del espacio de los parámetros [B de $\mathrm{R}$ ] contra [B de IRC] (similar al espacio del R-IRC de la Figura 1):

$$
B I R C_{I A F}=\alpha+\beta\left(B R_{I A F}\right)
$$

donde $\alpha$ y $\beta$ son los parámetros de la ecuación.
En el espacio meta-paramétrico $\alpha-\beta$ (espacio de parámetros), lineal, se puede modelar el patrón del crecimiento de la vegetación (incremento del IAF o cobertura aérea) con un modelo (similar al esquema de la Figura 2):

$$
\alpha=A A+B B \beta
$$

de tal forma que toda la información multi-angular asociada al sistema suelo-vegetación (efecto del suelo y del follaje) queda compactada en un espacio supraparamétrico (parámetros del espacio meta-paramétrico) AA-BB. De esta forma es posible analizar la viabilidad de discriminar clases de vegetación en forma compacta.

\section{Experimentos Bajo Condiciones Controladas de Laboratorio}

La discriminación de clases de vegetación a través de información espectral multi-angular se analizó mediante un experimento bajo condiciones controladas de laboratorio, en donde se simularon diferentes configuraciones geométricas de especies vegetales con arquetipos contrastantes. Para esto se utilizaron plantas pequeñas en las especies arbóreas y arbustivas $\mathrm{y}$, plantas adultas, para pastizales, después de podarse. En el Cuadro 1 se muestran las características principales de las plantas que se emplearon en los experimentos, en donde se consideran especies con diferentes tipos de hoja.

Las plantas individuales de cada especie, con mínimo estrés, se colocaron en bolsas de plástico y se acomodaron en tres densidades de acuerdo con

\begin{tabular}{|c|c|c|c|c|c|}
\hline Nombre científico & Nombre común & Tipo de hoja & Altura $^{\dagger}$ & Diámetro del tallo ${ }^{\dagger}$ & Diámetro del dosel \\
\hline & & & \multicolumn{3}{|c|}{ - - - - - - - - c cm- - - - - - - - } \\
\hline Abies religiosa & Oyamel & Aciculada & $34.34(5.33)$ & $0.70(0.14)$ & $24.26(6.44)$ \\
\hline Acacia retinodes & Mimosa & Ancha & $38.88(7.80)$ & $0.64(0.15)$ & $26.09(6.17)$ \\
\hline Casuarina equisetifolia & Casuarina & Aciculada & $36.67(3.85)$ & $0.64(0.17)$ & $23.87(5.97)$ \\
\hline Quercus rugosa & Encino & Ancha & $33.83(6.45)$ & $0.60(0.27)$ & $20.17(5.05)$ \\
\hline Fraxinus uhdei & Fresno & Ancha & $31.08(5.11)$ & $0.60(0.14)$ & $20.74(4.30)$ \\
\hline Eysenhardtia polystachya & Palo dulce & Ancha - micro & $25.29(10.7)$ & $1.99(1.42)$ & $15.90(5.17)$ \\
\hline Pinus greggii & Pino & Aciculada & $33.49(4.35)$ & $0.60(0.09)$ & $14.13(2.29)$ \\
\hline Bouteloa gracilis & Navajita & Lineal & $18.31(3.20)$ & $2.55(1.17)$ & $14.02(4.73)$ \\
\hline
\end{tabular}

Cuadro 1. Características de las especies vegetales usadas en el experimento.

${ }^{\dagger}$ Los datos entre paréntesis son las desviaciones estándar. 


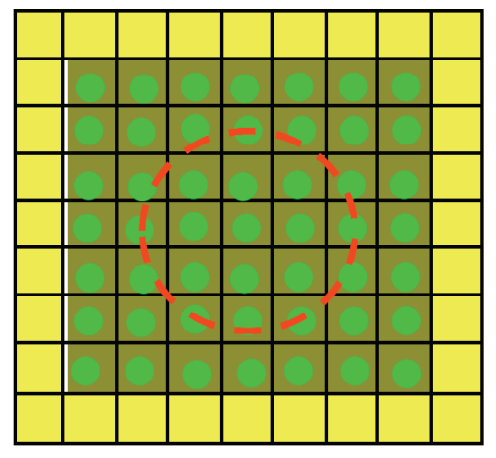

a

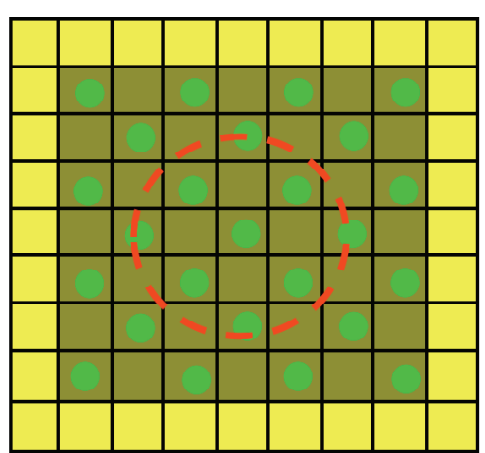

b

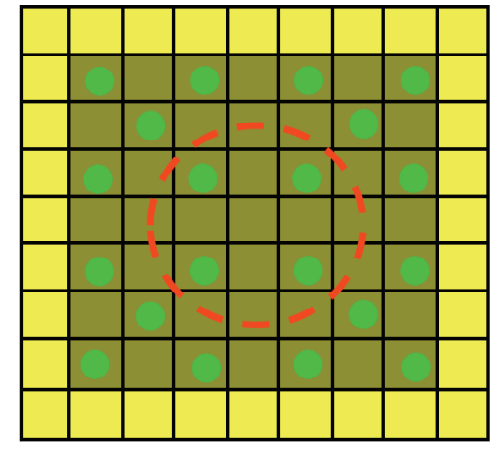

c

Figura 3. Simulaciones de configuraciones geométricas. Densidades usadas: a) alta, 49 plantas, b) media, 25 plantas y c) baja, 12 plantas. Cada círculo relleno representa la posición de una planta y los cuadros perimetrales más claros solo contienen suelo. El círculo al centro de los cuadros corresponde al área de visión del sensor hiperespectral con un ángulo de visión a nadir.

los arreglos que se muestran en la Figura 3. Los arreglos de plantas se colocaron en un cajón de madera de $1.5 \times 1.5 \mathrm{~m}$, con un sistema de mallas de $15 \times 15 \mathrm{~cm}$, para la colocación de las plantas individuales en bolsas de plástico, de tal manera que no se inclinaran al apoyarse sobre el fondo del cajón.

Para analizar el efecto del fondo de los arreglos de plantas, arriba de la malla superior del cajón se colocaron tiras de madera a la altura del inicio de los tallos de las plantas y sobre estas tiras, selladas en los tallos, se colocó suelo previamente seleccionado y con una granulometría menor a $2 \mathrm{~mm}$.

El cajón de madera con los arreglos de plantas forma parte del sistema goniométrico (SIGO-CP) de medición de reflectancias multi-angulares que se diseñó (Cano et al., 2014). A nadir, el radiómetro hiperespectral (350 a $2500 \mathrm{~nm}$ ) usado (modelo FR Jr de $\mathrm{ASD}^{\mathrm{MR}}$ ) mide un área circular de diámetro $0.41 \mathrm{~m}$. El radiómetro tiene un campo instantáneo de visión de $25^{\circ}$. Adicionalmente al radiómetro, se montaron en el SIGO-CP una cámara fotográfica digital (Cybershot DSC-V1 de Sony ${ }^{\mathrm{MR}}$ ) y un termómetro de radiación (modelo ITRS de Apogee ${ }^{\mathrm{MR}}$ ); instrumentos que se usaron para realizar mediciones cenitales a intervalos de $10^{\circ}$, desde $0^{\circ}$ a $60^{\circ}$, en las direcciones acimutales de $0^{\circ}$ a $350^{\circ}$, con intervalos de $10^{\circ}$. La dirección acimutal de $0-360^{\circ}$ de medición coincide con el acimut de la fuente de iluminación, por lo que las direcciones acimutales representan, en realidad, diferencias acimutales en la convención de $0-360^{\circ}$. La fuente de iluminación utilizada fue un reflector de aluminio de cuerpo negro de la marca Tecno Lite ${ }^{\mathrm{MR}}$ equipado con una lámpara de halógeno de cuarzo de 500 watts de la marca Osram ${ }^{\mathrm{MR}}$ (modelo 64706). Los filamentos de estas lámparas son de tungsteno y el vidrio que recubre el filamento está hecho de cuarzo, siendo el halógeno el gas que rellena el tubo o bombilla. La lámpara se montó en una base telescópica elevada a $3 \mathrm{~m}$ y su ángulo cenital $(\theta \mathrm{s})$ fue de $38^{\circ}$. Estas condiciones de iluminación se mantuvieron constantes para todas las mediciones de las especies vegetales analizadas.

Los suelos se colocaron sobre las tiras de madera a la altura del inicio de los tallos de las plantas, con un espesor de $1 \mathrm{~cm}$. En cada suelo se consideraron dos condiciones de humedad: suelo secado al aire y suelo húmedo producto de un evento de precipitación. Para este último caso se utilizó un simulador de lluvias tipo Morin (Morin et al., 1967), donde el cajón del SIGOCP con el arreglo de plantas se transportó a la base del simulador en el área experimental y se le aplicó un evento de precipitación con un disco de $75 \mathrm{~mm}$ durante 5 minutos con la llave del simulador abierta al máximo, lo cual aportó una lámina de precipitación de $8.3 \mathrm{~mm}$. El evento de precipitación se utilizó para analizar el efecto de la lluvia en el suelo del fondo de la vegetación, inmediatamente después del evento. La reflectancia del suelo después de aplicar un evento de precipitación se incrementó en proporción a la energía de las gotas de lluvia (Goldshleger et al., 2002). Adicionalmente, tal como se discute más adelante, el agua residual en las hojas de las plantas introduce errores en las mediciones del sistema suelo-vegetación, por lo cual los patrones de su BRDF resultan más erráticos que los de suelo secos.

En la Figura 4 se muestra la geometría de medición para el radiómetro montado en el arco cenital del SIGO- 

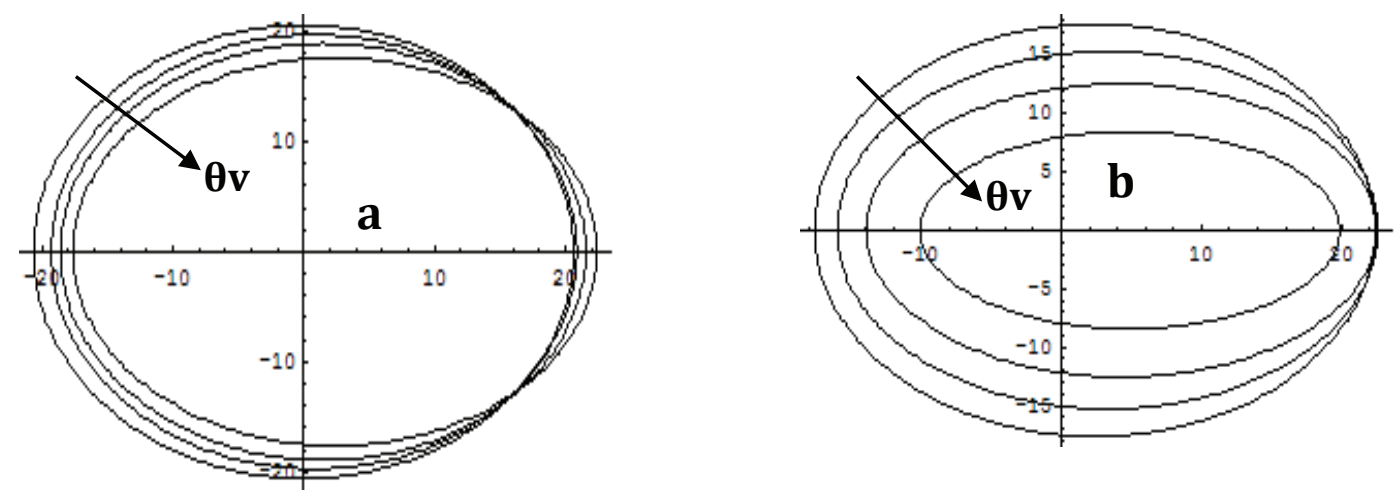

Figura 4. Áreas de visión del SIGO-CP a nadir: a) $\theta v$ de $0^{\circ}$ a $30^{\circ}$ y b) $\theta v$ de $30^{\circ}$ a $60^{\circ}$.

CP (Cano et al., 2014), en donde el área de visión para ángulos cenitales, no de nadir, forma una elipse cuyo centro está desplazado del centro del cajón.

En términos de la definición de la BRDF (Nicodemus et al., 1977), ésta implica ángulos sólidos infinitesimales por lo que no puede medirse en la práctica. El arreglo de medición de reflectancias en condiciones de laboratorio, que se discutió anteriormente, implica la caracterización de los factores de reflectancia bi-cónicos o cónico-cónico (CCRF por sus siglas en inglés) (Nicodemus et al., 1977; Schaepman-Strub et al., 2006), donde el termino cónico se usa para el ángulo sólido (iluminación y visión). Las mediciones de reflectancia del radiómetro hiperespectral se refieren en realidad a factores de reflectancia, los cuales se calculan en función de las propiedades ópticas de un panel de referencia (Hatchell, 1999).

El área del experimento se aisló con paños de tela negra y el techo se pintó de color negro para evitar contribuciones de radiación difusa y aproximar una fuente de iluminación con un ángulo cónico de solo radiación directa.

Ahora bien, de la Figura 3, la CCRF medida incorpora factores de escala (áreas de medición diferentes en función del ángulo cenital de visión), por lo que se debe usar una definición generalizada, en la que se considere la parte espacial del área de medición (Di Girolamo, 2003). Los cambios en las dimensiones de los píxeles con los ángulos de visión se presentan en los sensores AVHRR y MODIS, por lo que es importante considerar en forma explícita esta situación. En este caso, al considerar una relación exponencial entre las reflectancias y los IAF (Ross, 1981) como elementos de escala, se utilizó una nueva definición de reflectancias normalizadas: $\mathrm{Rn}=\ln (\mathrm{R})$ $\cos (\chi)$, ecuaciones (1), dejando igual el modelo de la BRDF (CCRF).

El procedimiento general de determinación de los factores de reflectancia, para cada especie en particular, sigue protocolos estándar de mediciones radiométricas.

Adicionalmente, se realizaron conjuntos completos de mediciones para el caso de suelo desnudo (suelos claro y oscuro, en condiciones secas y mojadas al aplicar un evento de precipitación con el simulador de lluvias). En cada conjunto de medición del arreglo de plantas de las especies se tomaron fotografías digitales para estimar la cobertura aérea (COB) mediante un proceso de clasificación supervisada.

Finalmente, las mediciones de reflectancia que se generaron (archivo de 350 a $2500 \mathrm{~nm}$, remuestreado a intervalos de $2 \mathrm{~nm}$ ) se convirtieron a las reflectancias de las bandas del sensor Landsat 5, con las funciones de respuesta correspondientes (https://landsat.gsfc.nasa. gov/spectral-response-of-the-multispectral-scannersystem-in-band-band-average-relative-spectralresponse/). Las reflectancias para las bandas Landsat 5 son simplemente el promedio de las mediciones generadas multiplicadas por la función de respuesta de la banda. Las bandas del azul y del infrarrojo medio no se analizaron debido a problemas relacionados con efectos de la atmósfera del área del experimento (vapor de agua) y de estabilidad del radiómetro, por lo que en lo siguiente solo se muestran los resultados de las bandas del verde (V), rojo (R) e infrarrojo cercano (IRC).

Los datos generados en el experimento se guardaron en archivos etiquetados con 4 dígitos: primer dígito: especie vegetal, segundo dígito: densidad del arreglo de plantas; tercer dígito: color del suelo $\mathrm{y}$, cuarto dígito: humedad del suelo. El Cuadro 2 muestra 
Cuadro 2. Codificación de los archivos del experimento.

\begin{tabular}{|c|c|c|c|c|c|c|c|}
\hline Nombre común especie & Código & Densidad de plantas & Código & Color del suelo & Código & Humedad del suelo & Código \\
\hline Suelo & 0 & Nula & 0 & Claro & 1 & Seco & 1 \\
\hline Oyamel & 1 & Alta & 1 & Oscuro & 2 & Mojado & 2 \\
\hline Mimosa & 2 & Media & 2 & & & & \\
\hline Casuarina & 3 & Baja & 3 & & & & \\
\hline Encino & 4 & & & & & & \\
\hline Fresno & 5 & & & & & & \\
\hline Palo dulce & 6 & & & & & & \\
\hline Pino & 7 & & & & & & \\
\hline Navajita & 8 & & & & & & \\
\hline
\end{tabular}

la codificación, de tal manera que un código 2121 significa planta Mimosa, densidad alta, color oscuro y seco.

En el caso de las coberturas aéreas, el Cuadro 3 muestra las estimaciones que se realizaron en las fotografías digitales, en donde $\mathrm{COB}$ global se refiere a la cobertura de todo el cuadro de medición ( $\sin$ los cuadros del borde) del cajón del SIGO-CP y COB local nadir es la cobertura del área del círculo de medición del radiómetro a nadir $(41.2 \times 41.2 \mathrm{~cm})$.

\section{RESULTADOS Y DISCUSIÓN}

\section{Ajuste de los Modelos de Ángulos Cenitales y Acimutales}

En la Figura 5 se muestran los ajustes del modelo de los ángulos cenitales de visión e iluminación para las reflectancias de las bandas del verde (V), rojo (R) e infrarrojo cercano (IRC) para las ocho especies, más el suelo desnudo. El error relativo medio general fue de $6.8 \%$, aceptable si se considera la diversidad de especies analizadas. El error relativo medio (ERM), en porcentaje, está definido por:

$E R M=\left\{\frac{1}{\mathrm{n}} \sum_{\mathrm{i}=1}^{\mathrm{n}}\left[\frac{R \text { med }- \text { Rest }}{R \text { med }}\right]\right\} \mathrm{x} 100$

donde med se refiere a medida, est a estimada y $\mathrm{R}$ a reflectancia de la banda del V, R e IRC.

La Figura 6 muestra un diagrama acimutal de los parámetros b para las bandas del R e IRC, sin considerar las diferencias acimutales de $0^{\circ}$ y $180^{\circ}$, por problemas
Cuadro 3. Coberturas aéreas asociadas a las densidades de plantas.

\begin{tabular}{|c|c|c|c|c|}
\hline Código & $\begin{array}{l}\text { Nombre común } \\
\text { especie }\end{array}$ & Densidad & $\begin{array}{c}\text { COB } \\
\text { Global }\end{array}$ & $\begin{array}{l}\text { COB local } \\
\text { nadir }\end{array}$ \\
\hline & & & \multicolumn{2}{|c|}{$-\ldots \%-\ldots$} \\
\hline 11 & Oyamel & Alta & 60.9 & 71.2 \\
\hline 12 & Oyamel & Media & 37.1 & 56.3 \\
\hline 13 & Oyamel & Baja & 25.0 & 28.2 \\
\hline 21 & Mimosa & Alta & 56.2 & 65.5 \\
\hline 22 & Mimosa & Media & 36.6 & 38.0 \\
\hline 23 & Mimosa & Baja & 25.7 & 24.1 \\
\hline 31 & Casuarina & Alta & 71.3 & 50.1 \\
\hline 32 & Casuarina & Media & 30.6 & 33.5 \\
\hline 33 & Casuarina & Baja & 25.5 & 18.0 \\
\hline 41 & Encino & Alta & 74.0 & 68.8 \\
\hline 42 & Encino & Media & 43.0 & 45.2 \\
\hline 43 & Encino & Baja & 31.2 & 27.7 \\
\hline 51 & Fresno & Alta & 60.8 & 61.3 \\
\hline 52 & Fresno & Media & 37.3 & 40.4 \\
\hline 53 & Fresno & Baja & 21.7 & 25.3 \\
\hline 61 & Palo Dulce & Alta & 45.7 & 41.3 \\
\hline 62 & Palo Dulce & Media & 28.6 & 21.0 \\
\hline 63 & Palo Dulce & Baja & 20.7 & 11.0 \\
\hline 71 & Pino & Alta & 53.1 & 55.6 \\
\hline 72 & Pino & Media & 23.3 & 26.9 \\
\hline 73 & Pino & Baja & 12.2 & 11.4 \\
\hline 81 & Navajita & Alta & 26.6 & 44.3 \\
\hline 82 & Navajita & Media & 30.5 & 30.2 \\
\hline 83 & Navajita & Baja & 18.0 & 18.4 \\
\hline 0 & Suelo & Nula & 0.0 & 0.0 \\
\hline
\end{tabular}




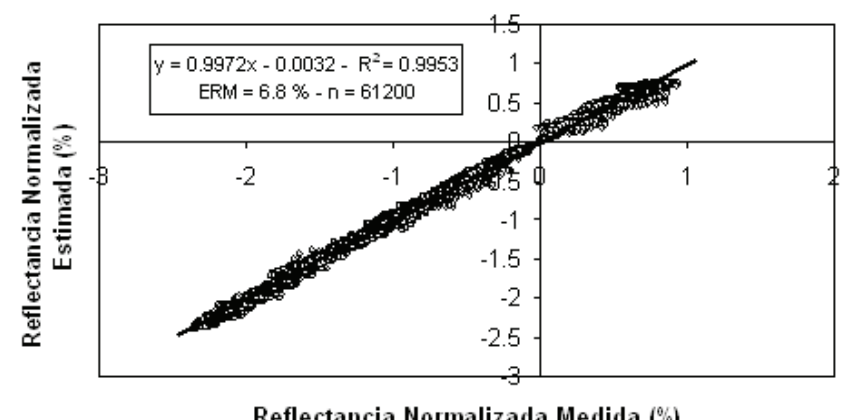

Reflectancia Normalizada Medida (\%)

Figura 5. Ajuste del modelo de los ángulos cenitales para las bandas del V, R e IRC.

de sombreado del área de visión por el goniométro, el caso de un suelo claro seco y mojado; para coberturas aéreas a nadir mayores $61 \%$. La Figura 7 muestra esta misma información para coberturas entre 45 y $55 \%$; y la Figura 8 para el caso de coberturas entre el 18 y $28 \%$.

Si se analizan los valores del parámetro $b$, se puede concluir, en lo general, que hay zonas de confusión (traslape) entre especies, que dependen de las propiedades ópticas de los suelos. Esto es particularmente crítico en las pendientes b de la banda del IRC, donde existe menor contraste entre los parámetros b. En el caso de la banda del R, el contraste es mayor; aunque mantiene los problemas de confusión en la discriminación de clases.

La Figura 9 muestra el peor ajuste del modelo de las diferencias acimutales, relaciones (2), que se refiere al caso de especie Encino con fondo de suelo oscuro y mojado, para la banda del V. En esa misma figura se muestra el ajuste del modelo para la banda del IRC (menos anisotrópico por efecto de interacciones múltiples de la radiancia). En lo general, los ajustes del modelo de las diferencias acimutales resulto en $\mathrm{R}^{2} \geq$ 0.99 .

\section{Ajuste de los Modelos del Efecto del Suelo y del Follaje}

En la Figura 10 se muestra las curvas iso-IAF multi-angulares (cuatro tipos de propiedades ópticas de los suelos del fondo de la vegetación), espacio multiangular del R-IRC, para el caso de la planta Mimosa (especie 2), en donde en cada curva se ajustó al modelo lineal de la relación (4) para su análisis en el espacio $\alpha-\beta$. En el caso del espacio multi-angular del R-IRC, los problemas de vegetación y suelo mojados se agudizan por tener patrones angulares sujetos a diferentes
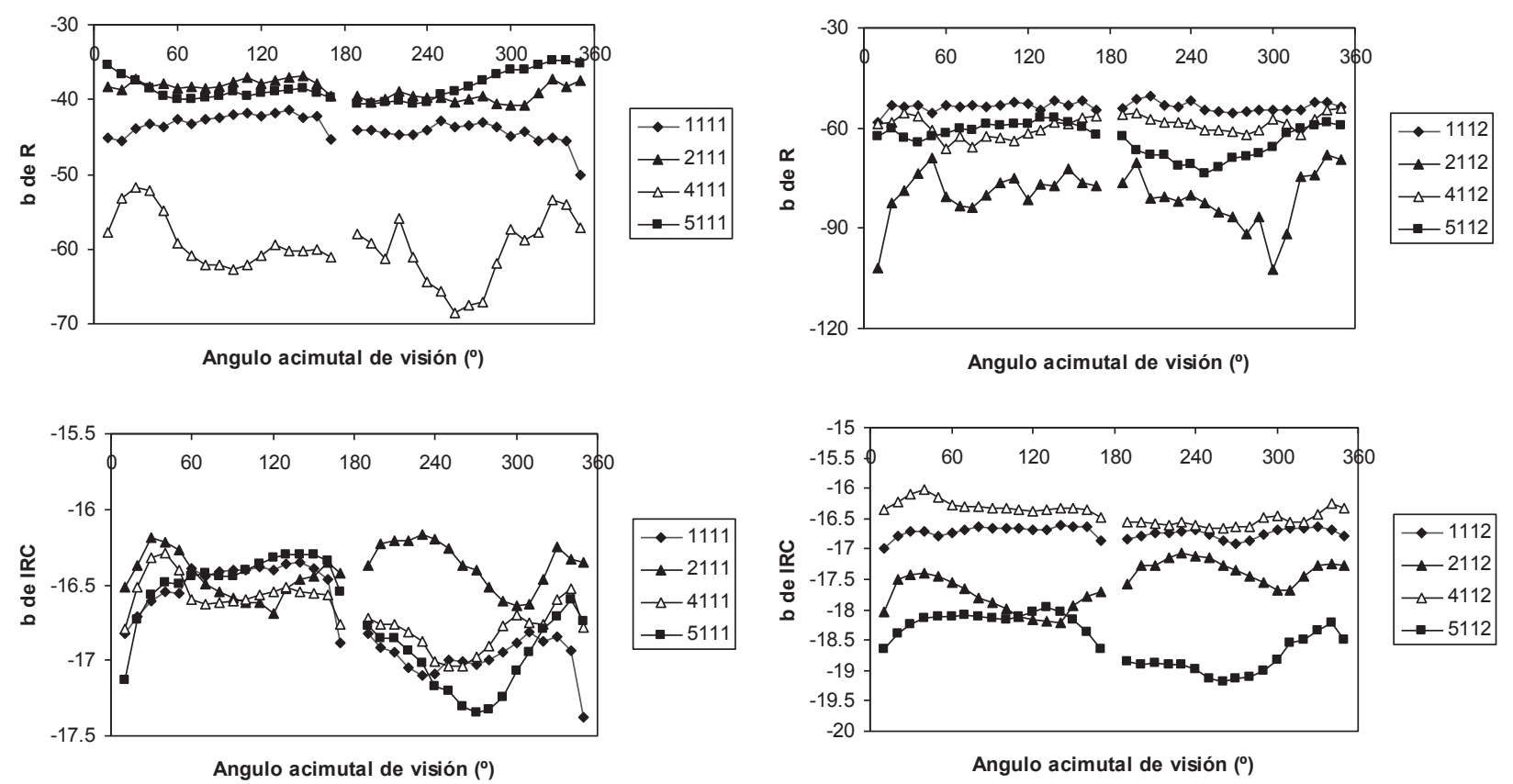

Figura 6. Diagramas acimutales de las bandas del R e IRC para un suelo claro seco y húmedo, con cobertura aérea a nadir mayor del $61 \%$. (Códigos mostrados en el Cuadro 2). 

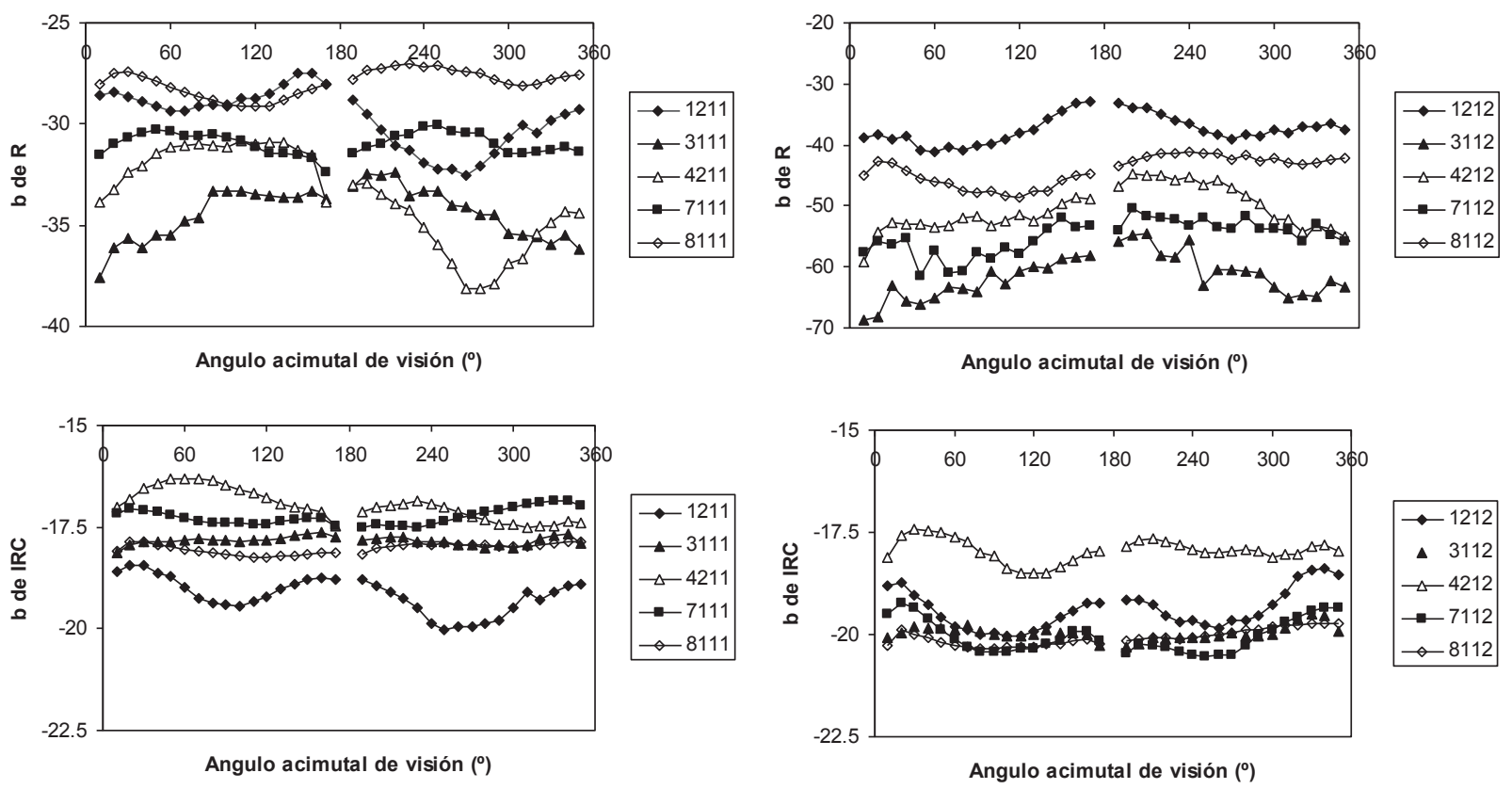

Figura 7. Diagramas acimutales de las bandas del $\mathrm{R}$ e IRC para un suelo claro seco y húmedo, con cobertura aérea a nadir entre el 45 y 55\%. (Códigos mostrados en el Cuadro 2).

tipos de interacción de la radiancia (predominio de las simples y las múltiples). Para reducir esa clase de efectos, se desecharon los datos de coberturas mayores al $60 \%$ para los casos de vegetación y suelo mojados.

En el caso de las bandas del visible, los efectos
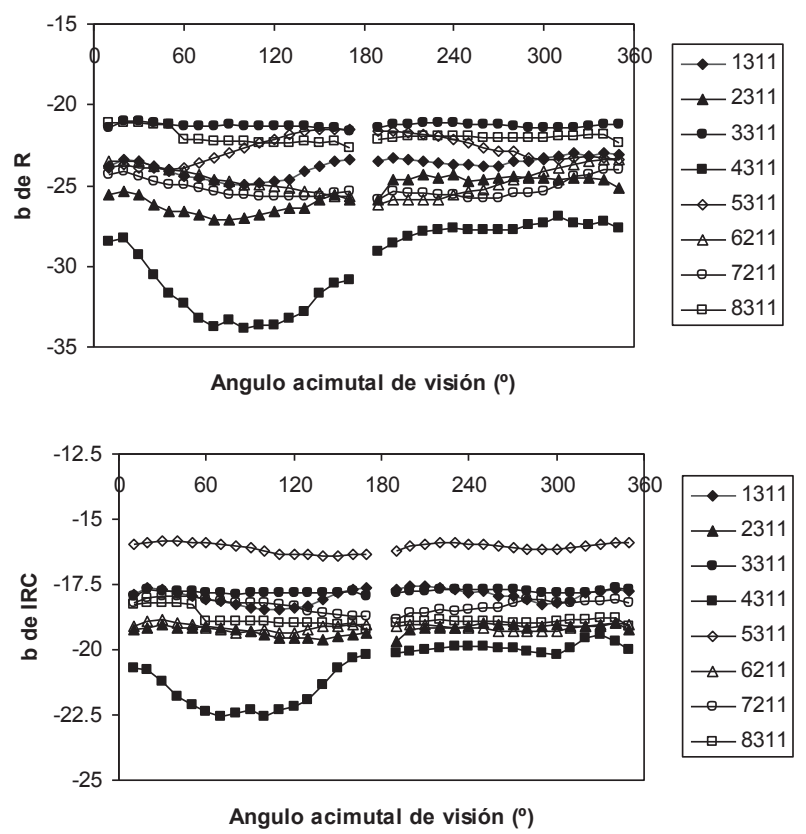

del mojado de la vegetación y suelo (interacciones simples) se presentan en todas las bandas, por lo que no es necesario eliminar datos con patrones diferentes. La Figura 11 muestra la situación de modelación para las bandas del V y del R.
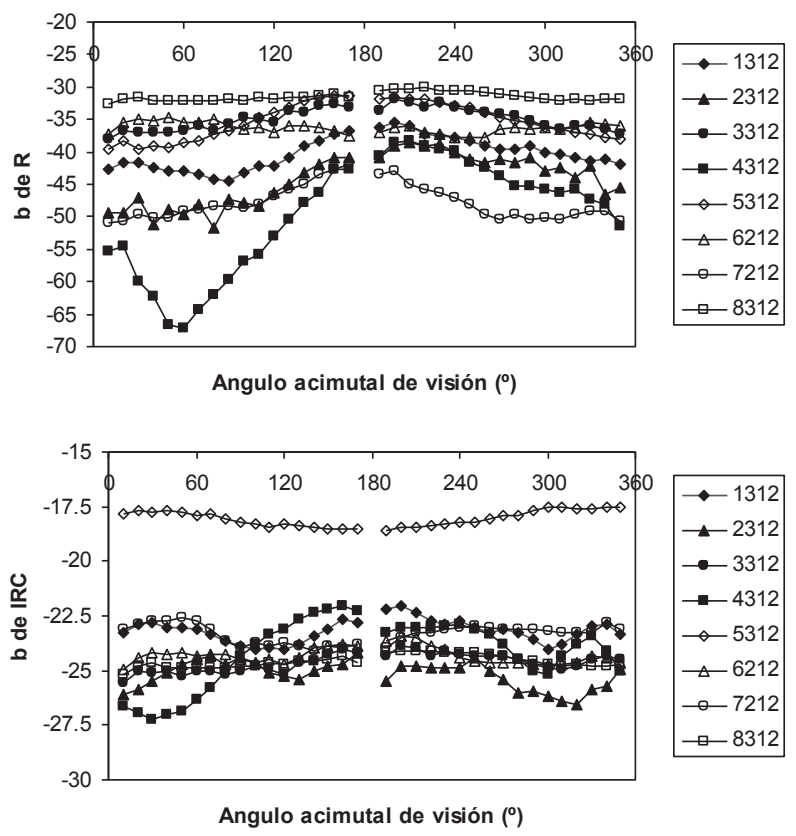

Figura 8. Diagramas acimutales de las bandas del R e IRC para un suelo claro seco y húmedo, con cobertura aérea a nadir entre el 18 y 28\%. (Códigos mostrados en el Cuadro 2). 

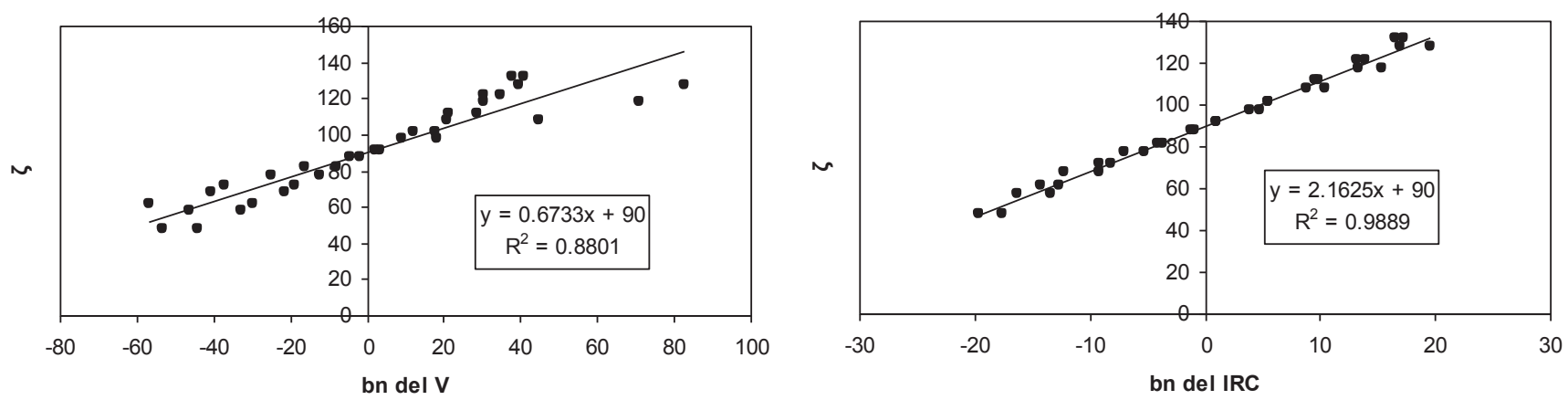

Figura 9. Peor ajuste del modelo de las diferencias acimutales para todas las especies y suelos.
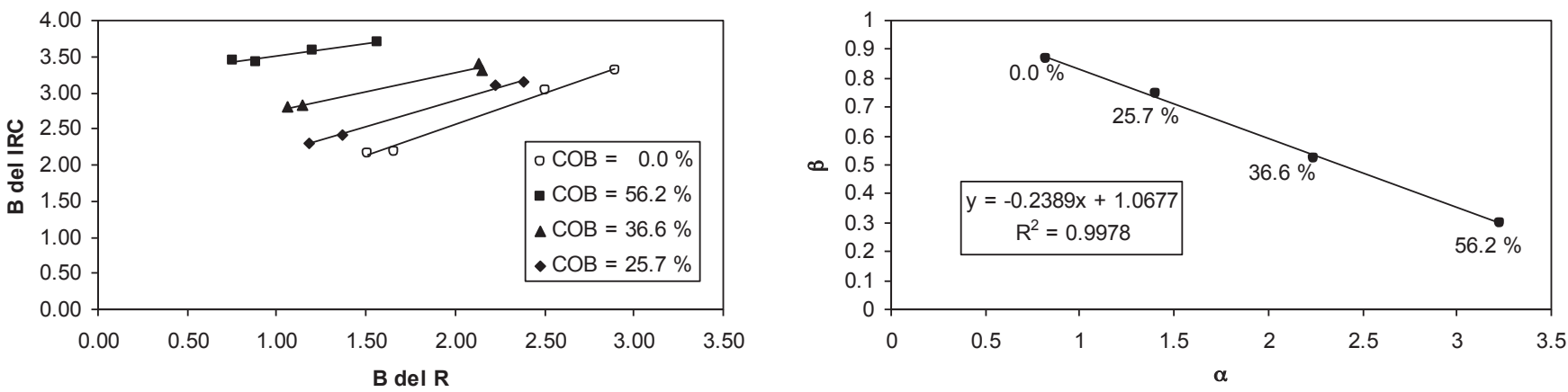

Figura 10. Patrones multi-angulares de los espacios B del R - B del IRC y $\alpha-\beta$ para la especie 2.
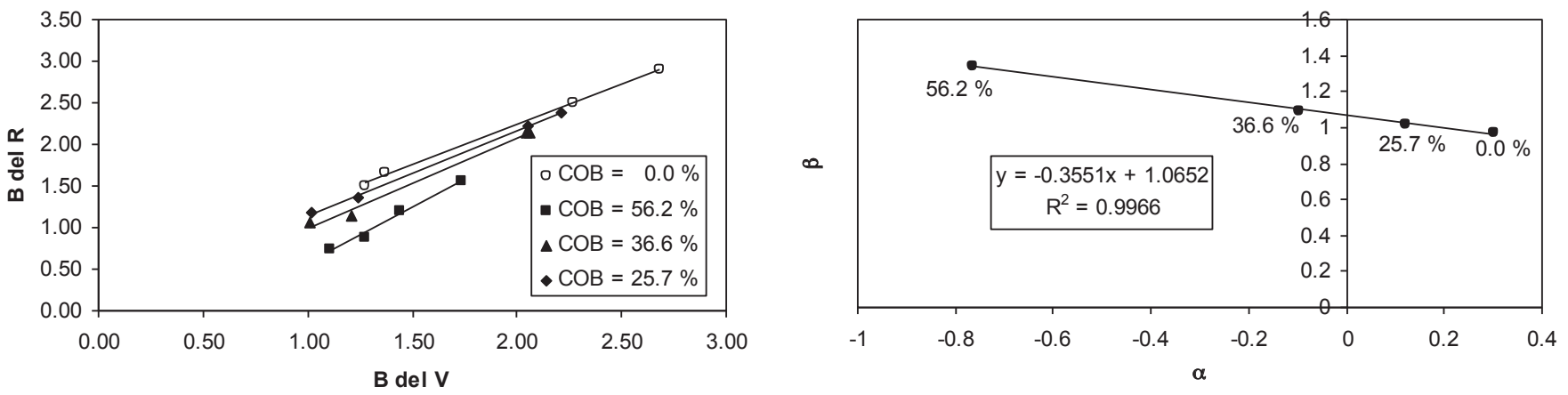

Figura 11. Patrones multi-angulares de los espacios B del V - B del R y $\alpha-\beta$ para la especie 2.

En lo general, los patrones ajustados del modelo de la relación (4) y (5) muestran valores de $\mathrm{R}^{2} \geq 0.99$. Así, la modelación de las mediciones multi-angulares de las reflectancias puede compactarse al caso de dos parámetros (AA y BB de la relación 5).

\section{Modelo Global Ajustado y Discriminación de Especies}

De acuerdo con la relación (5), en el espacio supra-paramétrico (parámetros de las relaciones meta- paramétricas) AA-BB se pueden sintetizar los efectos multi-angulares del suelo y del follaje. El uso de este espacio permite analizar la posible discriminación de las especies, sujeto a variaciones del fondo de suelo, geometría sol-sensor y cobertura del follaje.

En la Figura 12 se muestra el espacio AA-BB para el caso de los patrones asociados a las bandas del $\mathrm{R} \mathrm{e}$ IRC y en la Figura 13 la de las bandas del V y R.

Aunque las Figuras 12 y 13 muestran patrones separables de la información multi-angular para las distintas especies analizadas, los valores de los supra- 


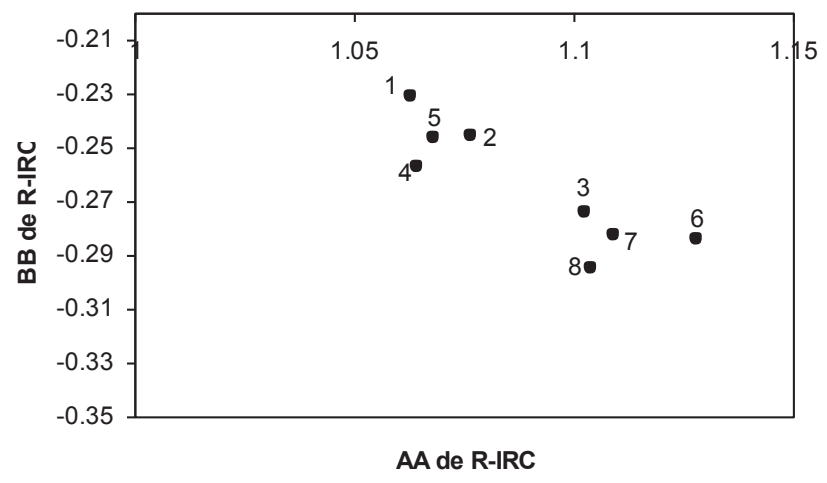

Figura 12. Espacio supra-paramétrico AA-BB para las bandas del R e IRC. (Códigos mostrados en el Cuadro 2).

parámetros $\mathrm{AA}$ y BB están muy cercanos entre sí, por lo que las clases de vegetación resultan difíciles de discriminar en términos operativos.

Por otro lado, las posiciones de los patrones AA-BB para las distintas especies no muestran una coherencia (en apariencia) con relación a las propiedades ópticas $\mathrm{y}$ angulares del follaje de las especies analizadas (Cuadro 1).

\section{CONCLUSIONES}

- Los resultados muestran que las clases de vegetación analizadas resultan en confusión (traslapes), por lo que la información multi-angular tiene una contribución relativamente baja para discriminar entre especies, esto bajo la perspectiva de considerar simultáneamente geometría sol-sensor, propiedades ópticas diferentes de los suelos debajo de la vegetación y condiciones de humedad diferente, así como coberturas (densidades) del follaje. Estas consideraciones difieren de los estudios clásicos de discriminación que consideran en lo general uno o dos factores.

- La conclusión aparente de discriminaciones relativamente pobres en el espacio supra-paramétrico propuesto está basada en las distancias pequeñas entre los puntos que representan a las especies. No obstante, esta situación, es necesario enfatizar que cada punto representa múltiples variaciones de los factores analizados, por lo que la posible confusión en las discriminaciones en función de distancias pequeñas de separación de las clases requiere de una revisión bajo la óptica de definir, en términos operativos, las implicaciones de estas distancias.

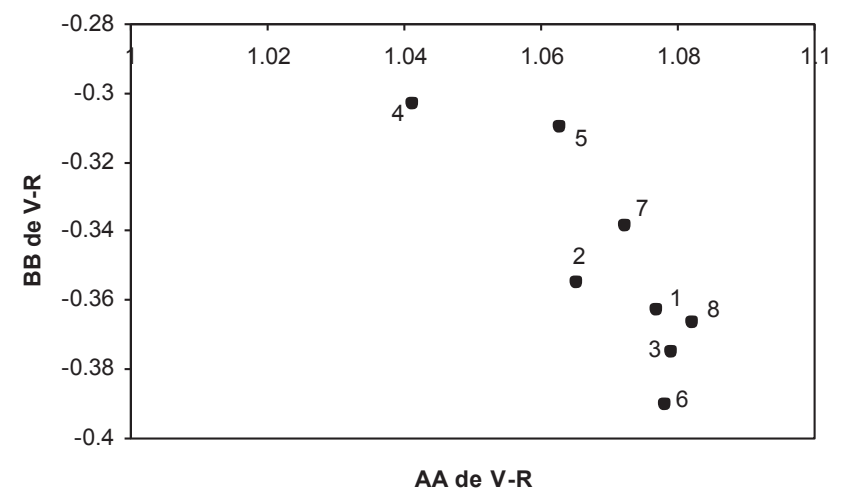

Figura 13. Espacio supra-paramétrico AA-BB para las bandas del V y R. (Códigos mostrados en el Cuadro 2).

- Es necesario realizar otros tipos de modelaciones: relación entre los parámetros $\mathrm{B}$ y las reflectancias a nadir y efecto multi-angular en las curvas iso-IAF de acuerdo con el esquema planteado en Paz et al. (2007). No obstante, esto, el hecho de modelar en forma completa los efectos multi-angulares de las reflectancias con un solo parámetro $(\mathrm{B})$, requiriendo un solo dato (aplicable a nivel pixeles individuales, sin ninguna hipótesis de homogeneidad o estacionariedad), plantea un camino para fusionar información multi-angular con la determinada a nadir por satélites no-angulares.

\section{LITERATURA CITADA}

Asner, G. P. 2000. Contributions of multi-view angle remote sensing to land-surface and biogeochemical research. Remote Sens. Rev. 18: 137-162.

Barnsley, M. J., D. Allison, and P. Lewis. 1997. On the information content of multiple view angle (MVA) images. Int. J. Remote Sens. 18: 1937-1960.

Bicheron, P., M. Leroy, O. Hautecoeur, and F. M. Bréon. 1997. Enhanced discrimination of boreal forest covers with direccional reflectances from the airborne polarization and directionality of earth reflectances (POLDER) instrument. J. Geophys. Res. 102: 29517-29528.

Bolaños, M., F. Paz, E. Palacios, E. Mejía y A. Huete. 2007. Modelación de los efectos de la geometría sol-sensor en la reflectancia de la vegetación. Agrociencia 41: 527-537.

Bolaños, M. y F. Paz. 2010. Modelación general de los efectos de la geometría iluminación-visión en la reflectancia de pastizales. Rev. Mex. Cienc. Pecu. 1: 349-361.

Cano, A., F. Paz, A. Zarco, M. I. Marín, E. López, J. Chávez, M. Bolaños y J. L. Oropeza. 2014. Diseño de un sistema goniométrico con tres grados de libertad para medir reflectancias en el sistema suelo-vegetación. Terrra Latinoamericana 32: 59-68. 
Di Girolamo, L. 2003. Generalizing the definition of the bidirectional reflectance distribution function. Remote Sens. Environ. 88: 479-482.

Diner, D. J., B. H. Braswell, R. Davies, N. Gobron, J. Hu, Y. Jin, R. A. Khan, Y. Knyazikhin, N. Loeb, J. P. Muller, A. W. Nolin, B. Pinty, C. B. Schaaf, G. Seiz, and J. Stroeve. 2005. The value of multiangle measurements for retrieving structurally and radiatively consistent properties of clouds, aerosols, and surfaces. Remote Sens. Environ. 97: 495-518.

Diner, D. J., G. P. Asner, R. Davies, Y. Knyazhikin, J. P. Muller, A.W. Nolin, B. Pinty, C. B. Schaaf, and J. Stroeve. 1999. New directions in earth observing: Scientific applications of multiangle remote sensing. Bull. Metereol. Soc. 80: 2209-228.

Engelson, O., B. Pinty, M. M. Verstraete, and J. V. Martonchik. 1996. Parametric bidirectional reflectance factor models: Evaluation, improvements and applications. Joint Research Centre (Europan Comission). Catalogue: CL-NA16426-EN-C. Ispra, Italy.

Gao, F., C. B. Schaaf, A. H. Strhler, Y. Jin, and X. Li. 2003. Detecting vegetation structure using kernel-based BRDF model. Remote Sens. Environ. 86: 198-205.

Gerard, F. 2003. Single angle, dual angle and multi-temporal viewing: Assessing through modelling the implications for forest structure variable extraction. Int. J. Remote Sens. 24: 1317-1334.

Gerard, F. F. and P. R. J. North. 1997. Analyzing the effect of structural variability and canopy gaps on forest BRDF using a geometric-optical model. Remote Sens. Environ. 62: 46-63.

Gobron N., B. Pinty, M.M. Verstraete, J. L. Widlowski, and D. J. Diner. 2002. Uniqueness of multiangular measurements. II: Joint retrieval of vegetation structure and photosynthetic activity from MISR. IEEE Trans. Geosci. Remote Sens. 40: 1574-1592.

Goldshleger, N., E. Ben-Dor, Y. Benyamini, D. Blumberg, and M. Agassi. 2002. Spectral properties and hydraulic conductance of soil crusts formed by raindrop impact. Int. J. Remote Sens. 23: 3909-3920.

Hatchell, D. C. 1999. Analytical Spectral Devices, Inc. Technical guide. Boulder, $\mathrm{CO}$, USA.

Hyman, A. H. and M. J. Barnsley. 1997. On the potential for land cover mapping from multiple-view-angle (MVA) remotelysensed images. Int. J. Remote Sens. 18: 2471-2475.

Knyazikhin, Y., J. V. Martonchik, D. J. Diner, R. B. Myneni, M. Verstraete, B. Pinty, and N. Gobron. 1998a. Estimation of vegetation canopy leaf area index and fraction of absorbed photosynthetically active radiation from atmosphere-correct MISR data. Theory J. Gephys. Res. 103: 32239-32256.

Knyazikhin, Y., J. V. Martonchik, R. B. Myneni, D. J. Diner, and S. W. Running. 1998b. Synergistic algorithm for estimating vegetation canopy leaf area index and fraction of absorbed photosynthetically active radiation from MODIS and MISR data. Theory J. Gephys. Res. 103: 32257-32275.
Lotsch, A., Y. Tian, M.A. Friedl, and R. B. Myneni. 2000. Land cover mapping in support of LAI and FAPAR retrievals from EOS-MODIS and MISR: Classification methods and sensitivities to errors. Int. J. Remote Sens. 24: 1997-2016.

Lovell, J. L. and R. D. Graetz. 2002. Analysis of POLDER-ADEOS data for the Australian continent: The relationship between BRDF and vegetation structure. Int. J. Remote Sens. 23: 27672796.

Morin, J., D. Goldberg, and I. Seginer. 1967. A Rainfall simulator with a rotating disk. Trans. ASAE 10: 74-79.

Myneni, R. B., R. Ramakrishna, R. R. Nemani, and S. W. Running. 1997. Estimation of global leaf area index and absorbed PAR using radiative transfer models. IEEE Trans. Geosci. Remote Sens. 35: 1380-1393.

Nicodemus, F. E., J. C. Richmond, J. J. Hsia, I. W. Ginsberg, and T. Limperis. 1977. Geometrical considerations and nomenclature for reflectance. NBS Monograph 160. U. S. Department of Commerce. Washington, DC, USA.

Paz, F., E. Palacios, E. Mejía, M. Martínez y L. A. Palacios. 2005. Análisis de los espacios espectrales de la reflectividad del follaje de los cultivos. Agrociencia 39: 293-301.

Paz, F., E. Palacios, M. Bolaños, L.A. Palacios, M. Martínez, E. Mejía y A. Huete. 2007. Diseño de un índice espectral de la vegetación: NDVIcp. Agrociencia 41: 539-554.

Paz, F. y E. Medrano. 2015. Patrones espectrales multi-angulares de clases globales de coberturas del suelo usando el sensor remoto POLDER-1. Terra Latinoamericana 33: 129-137.

Pinty B., J. L. Widlowski, N. Gobron, M. M. Verstraete, and D. J. Diner. 2002. Uniqueness of multiangular measurements. I. An indicator of subpixel surface heterogeneity form MISR. IEEE Transactions on Geoscience and Remote Sensing 40: 15601573.

Rahman, H., B. Pinty, and M. M. Verstraete. 1993. Coupled surfaceatmosphere reflectance (CSAR) model: 2. Semiempirical surfaces model usable with NOAA advanced very high resolution radiometer data. J. Geophys. Res. 98: 20791-20801.

Ross, J. K. 1981. The radiation regime and architecture of plant stands. Dr. W. Junk. Hingham, MA, USA.

Schaepman-Strub, G., M. E. Schaepman, T. H. Painter, S. Dangel, and J. V. Martonchik. 2006. Reflectance quantities in optical remote sensing - definitions and case studies. Remote Sens. Environ. 103: 27-42

Wanner W., X. Li, and A. H. Strahler. 1995. On the derivation of kernels for kernel-driven models of bidirectional reflectance. J. Geophys. Res. 100: 21077-21089.

Zhang Y., Y. Tian, R. B. Myneni, and Y. Knyazikhin. 2002a. Required consistency between biome definitions and signatures with the physics of remote sensing. I: Empirical arguments. Remote Sens. Environ. 80: 418-434.

Zhang, Y., N. Shabanov, Y. Knyazikhin, and R. B. Myneni. 2002b. Required consistency between biome definitions and signatures with the physics of remote sensing. II: Theoretical arguments. Remote Sens. Environ. 80: 435-446. 